\title{
Análisis contingencial: una propuesta de intervención en un caso clínico
}

\author{
Junior Gómez-Gonzales ${ }^{1}$
}

RESUMEN: En el presente artículo se diseña una propuesta de intervención psicológica de acuerdo a la metodología del análisis contingencial, desde el enfoque interconductual, considerando el caso clínico de un adolescente de trece años que acude al servicio de atención psicológica en una institución hospitalaria de Lima-Perú. Se presentan además las bases teóricas fundamentales del modelo contingencial y se diseña un esquema de intervención a partir de los datos del caso considerando, a su vez, el uso de técnicas conductuales adaptadas a este enfoque. Este método se constituye como una válida alternativa de diagnóstico, evaluación e intervención psicológica en el ámbito clínico.

PALABRAS CLAVES:

Citar como:
Análisis contingencial; Enfoque interconductual; Caso clínico; Adolescencia.

Gómez J. Análisis contingencial: una propuesta de intervención en un caso clínico. CASUS. 2016; 1(1):34-40. 


\section{INTRODUCCIÓN}

El análisis contingencial es una metodología de aplicación psicológica utilizada para el cambio del comportamiento humano individual que involucra el análisis de la valoración positiva o negativa que hace la persona en relación a las conductas y creencias que se dan en una interacción (1). El objetivo de esta metodología es abordar la situación problemática de un paciente a partir del enfoque interconductual (1). El objeto de estudio de este enfoque es: "la interacción del organismo total con su medio ambiente físico, biológico y/o social" (2). Esta metodología se presenta como una alternativa de aplicación para la labor que realiza el psicólogo. La misma ha mostrado resultados favorables en la intervención clínica $(3,4)$

El análisis contingencial, de acuerdo a lo que plantean Rodríguez y Landa, tiene un método que consta de cinco fases (1-3). La primera fase es la identificación de las relaciones microcontingenciales, referidas a todos los factores o circunstancias específicas en las que se desarrolla una interacción. Estas se pueden valorar como problema en la medida en que la persona o grupo social asumen de esta manera. En este sistema microcontingencial se establecen cuatro categorías para analizar: morfologías de la conducta (la acción que realiza la persona al relacionarse con el entorno), situaciones (factores del contexto o ambiente que permiten o interfieren en el desarrollo de la interacción problemática), comportamiento de otras personas (la función que cumplen las personas significativas para el paciente al realizar una acción en la interacción que se define como problema) y efectos (son las consecuencias de la interacción que puede alterar la conducta del paciente (1-3).

La segunda fase es el análisis macrocontingencial que permite evaluar la valoración que el paciente hace de las acciones o creencias de las personas o grupos con los que interactúa en donde se identifican dos tipos. La ejemplar que funciona como ejemplo de cómo debería ser la relación social y la situacional corresponde a la interacción que se valora como problema. La tercera fase es el análisis de la génesis o inicio del problema, referido a la identificación del origen histórico de una interacción problemática (1-3). Aquí se identifican los aspectos que son relevantes para explicar el problema y su correspondiente afectación a la conducta, asumiendo que esta explicación no es de tipo causal, sino que existen diferentes elementos que la producen.
La cuarta fase corresponde al análisis de soluciones, en la que se plantean con el paciente todas las posibles alternativas de cambio, tomando en cuenta la evaluación realizada en las tres fases anteriores. De acuerdo a ello se decide la posibilidad de incidir en el mantenimiento o cambio de las interacciones. Finalmente la quinta fase corresponde al diseño, selección, aplicación y evaluación de la intervención donde se elaboran los procedimientos de acuerdo a la evaluación realizada. Esta intervención se centra en los siguientes procedimientos: para alterar las disposiciones (factores que afectan la conducta actual), para alterar la conducta de otros, para alterar la propia conducta y para la alteración de prácticas macrocontingenciales. De acuerdo a estos procedimientos se aplican técnicas existentes adaptadas al enfoque interconductual, o por el contrario se elaboran nuevas con diversos propósitos funcionales.

A partir del enfoque interconductual se han realizado investigaciones con la finalidad de desarrollar y difundir el mismo. Entre ellas resaltan los aportes en el contexto educativo a través de la forma de observar el aprendizaje desde esta perspectiva (4-6). Por ejemplo, en el área educativa se han empleado las teorías planteadas para rediseñar las currículas de carreras profesionales (7). Igualmente destacan contribuciones a nivel organizacional (8). Partiendo de la propuesta de replantear el enfoque teórico en este campo se han diseñado esquemas para la identificación de competencias laborales desarrollado métodos para evaluarlas e identificarlas permitiendo una mejor intervención en esta área $(9,10)$. En el campo clínico se han obtenido resultados favorables con el procedimiento de Intervención Interconductual en el contexto clínico (IICC) (11) y en las intervenciones desarrolladas en casos únicos se han logrado los objetivos de intervención planteados $(3,4)$.

Teniendo en cuenta los elementos mencionados el presente artículo diseña una propuesta de intervención de acuerdo a esta metodología desarrollando el método del análisis contingencial, desde el enfoque interconductual, considerando el caso clínico de un adolescente de trece años que acude al servicio de atención psicológica en una institución hospitalaria en Lima. 


\section{PRESENTACIÓN DEL CASO}

\section{Datos de Filiación}

Se presenta un adolescente varón de 13 años, estudiante del 2 año de secundaria, acompañado por la madre que inicialmente refiere las dificultades por la cual acude a consulta.

\section{Motivo de consulta}

La madre refiere que su hijo está presentando dificultades relacionadas a las habilidades matemáticas desde que inició la educación secundaria. Además señala que no acata las órdenes vinculadas a las actividades en casa y que se muestra irascible. Por otra parte, el paciente manifiesta que la madre se enfada y demuestra una actitud muy exigente hacia él, lo cual origina sus problemas de interacción.

En las interacciones, el adolescente manifiesta tener dificultad para expresar y controlar sus emociones, sobre todo cuando está en situaciones que percibe como opuestas a lo que siente o piensa. Esto hace que en algunas ocasiones se abstenga de interactuar con los demás. Refiere sentirse preocupado por la relación con su madre, reconociendo que ella se ve afectada por esta situación. Igualmente señala sentirse mal por las dificultades académicas mencionadas y que últimamente la calidad de su sueño se ha visto afectada.

\section{Desarrollo cronológico del problema}

Según lo manifestado por el adolescente los problemas de interacción con la madre se iniciaron aproximadamente a los siete años cuando entró en conocimiento de que ella fue víctima de maltrato físico y abuso sexual por parte del padre biológico del paciente. Por esta razón actualmente presenta al recordar siente ira y no desea conocer a su padre, tanto menos hablar sobre este tema. Otro hecho significativo (nueve años) fue cuando el perro doméstico le mordió el rostro dejándole una notoria cicatriz, la cual es motivo de burla de algunos de sus compañeros de escuela. Debido a esto se torna agresivo con los mismos presentando posteriormente culpa porque valora que la forma en que actúa no es adecuada. Estas interacciones, en las que el adolescente frecuentemente prefiere no reaccionar "haciendo daño" a las personas debido a que no sabe cómo expresar su ira, se siguen presentando hasta la actualidad.
Por otro lado, en relación a la dificultad con las matemáticas, el paciente refiere que este problema se desarrolla cuando cursaba el grado de primaria. El mismo refiere que el método de enseñanza usado por sus profesores de primaria no permite que consolide los aprendizajes esperados en ese grado. En consecuencia cuando ingresa a primer grado de secundaria no logra el aprendizaje esperado en matemáticas. Actualmente está en segundo de secundaria y expresa que esta dificultad le afecta emocionalmente y en la interacción con los demás.

\section{Situación familiar}

El paciente vive con la madre y el padrastro. La madre de 33 años es contadora de profesión. En la relación que tiene con el paciente actualmente hay confianza y algunos altercados a nivel comunicacional que lo llevan a experimentar emociones de ira o tristeza. El padrastro de 41 años es quien en el hogar aporta económicamente. La relación que tiene con el paciente es de confianza y de soporte emocional, considerándolo como su padre.

\section{Evaluación y diagnóstico}

Según la metodología del análisis contingencial se plantea el siguiente esquema de evaluación e intervención para el caso expuesto. Se realiza el análisis de las contingencias (situaciones problemáticas) valoradas como problema. Para fines prácticos a partir de aquí se indicará a la contingencia 1 como Ay a la contingencia 2 como B.

\section{Análisis microcontingencial \\ Morfologías de la conducta del paciente:}

A:

-En ocasiones no expresa lo que siente cuando algo le fastidia y se queda callado.

-A veces no puede quedarse callado y grita a su madre o golpea algún objeto para calmar su ira.

B:

-Se enoja al no comprender y evita las actividades vinculadas a las matemáticas.

-Al sentirse obligado a concretar una actividad académica se enfada y reacciona gritando.

\section{Morfologías de los otros significativos: \\ De la madre:}

A:

- Se enfada cuando no obedece o no realiza las actividades. 
- Expresa tristeza y/o comenta situaciones pasadas con el paciente.

- Se preocupa por el paciente.

B:

- Le preocupa el rendimiento académico del paciente.

\section{Situaciones:}

Circunstancia Social. La circunstancia (según las microcontingencias) que se valora como problema es la relación con la madre y con los pares (A) y la escuela (B).

Conducta socialmente esperada del paciente:

A:

- Su familia espera que pueda expresar lo que piensa y siente controlando su ira.

B:

-La familia espera que mejore el rendimiento en el curso de matemática.

Capacidad en el ejercicio de dichas conductas:

A:

-Presenta dificultad para expresar las emociones, se abstiene o expresa ira.

B:

- Posee las habilidades para mejorar el rendimiento académico.

\section{Comportamiento de otras personas:}

Mediador (Persona o grupo de personas que permiten

o interfieren para que se presenten los comportamientos que generan la interacción problemática):

A:

El propio comportamiento que realiza el paciente está mediando la relación con el grupo de pares y el comportamiento de la madre está mediando la relación con el paciente.

B:

El comportamiento del docente para la enseñanza está mediando el comportamiento del paciente.

Mediado (Persona o grupo de personas que presentan los comportamientos que generan la interacción problemática):

A:

El comportamiento del grupo de pares está mediado en función del comportamiento del paciente y el comportamiento del usuario está mediado en función del comportamiento de la madre.

B:

El comportamiento del paciente en referencia al aprendizaje está mediado en función del comportamiento del docente.

Efectos:

Sobre sí mismo:

A:

-Siente frustración porque preferiría poder expresar lo que piensa y siente de manera asertiva.

-Luego de expresar ira experimenta tristeza y culpa por dañar a los demás.

B:

-No logra adecuarse a la enseñanza del docente y experimenta frustración.

\section{Sobre los otros:}

A:

- Preocupación de la madre y el padrastro por su comportamiento.

- El comportamiento y la actitud "pasiva" del paciente al relacionarse y al no expresar lo que siente y piensa hace que los otros determinen las características de la interacción actual.

\section{B:}

- La madre y el padrastro se preocupan por el rendimiento académico.

\section{Análisis macrocontingencial}

Según lo referido por el paciente y la madre:

La valoración personal que contextualiza el problema de expresión de emociones está relacionada con no generar violencia hacia los demás.

\section{Microcontingencia ejemplar}

Prácticas valorativas efectivas:

Madre del paciente: No generar violencia física ni psicológica a los demás.

Padrastro del paciente: No generar violencia física ni psicológica.

\section{Prácticas valorativas sustitutivas (creencias):}

Madre del paciente: Cree que generar violencia física y psicológica no es adecuado.

Padrastro del paciente: No se obtuvo la información. 


\section{Microcontingencia situacional}

Prácticas efectivas:

Paciente: Trata de no generar violencia hacia los demás, aunque algunas veces no puede controlar su ira y expresa violencia psicológica.

Madre del paciente: Genera violencia psicológica cuando se enfada y le grita al paciente.

Compañeros del paciente: Generan violencia psicológica cuando lo insultan por su cicatriz.

\section{Prácticas sustitutivas:}

Paciente: Cree que cuando no controla su ira está ejerciendo violencia psicológica hacia los demás.

Madre del Paciente: Cree que no debería enfadarse ni gritar a su hijo ya que no logra nada haciéndolo.

Compañeros del paciente: No se obtuvo la información.

Génesis del problema bajo el enfoque del análisis contingencial

Historia de la microcontingencia:

1. Circunstancia en que se inició la conducta problema: A. Relato de la madre sobre el maltrato que recibió del padre biológico.

B. Interacciones con el docente que tuvo de cuarto a sexto grado de primaria con respecto a los métodos y prácticas de enseñanza.

Situación en que se inició la microcontingencia actual:

A. La relación que mantiene el paciente actualmente con la madre y los compañeros se inicia cuando la madre empieza a enfadarse y gritarle al paciente.

B. A partir de la interacción con el docente de matemáticas en primer grado de secundaria.

Funcionalidad de las conductas manifestadas en otros contextos:

A. La funcionalidad de las conductas asociadas a la expresión y control de emociones se desarrolla en el contexto familiar y social.

B. La funcionalidad de las conductas asociadas al rendimiento académico se restringe al contexto educativo.

Disponibilidad de otras conductas no problemáticas potencialmente funcionales en la microcontingencia presente:

La capacidad del paciente de comprensión y el seguimiento de instrucciones de personas que considera capaz de ayudarlo fueron identificados como potencialmente funcionales para la solución del problema.

\section{Diseño}

Según el análisis contingencial corresponde la intervención a desarrollar el análisis de las soluciones que se realiza junto al paciente. Además la selección, diseño, aplicación y evaluación de las estrategias de intervención en la cual en el artículo solo se desarrollara la selección y el diseño de las estrategias del programa de intervención.

\section{Análisis de soluciones}

El análisis de soluciones es una propuesta que busca mantener o cambiar las microcontingencias y macrocontingencias descritas. En el análisis de soluciones para A el paciente decidió el cambio en la conducta propia para desarrollar habilidades que le permitan la expresión y el control de las emociones frente al entorno. A su vez se decidió incidir en el cambio de la conducta de otros, en este caso el cambio de conducta de la madre, ya que media el comportamiento del paciente. El cambio correspondiente permitirá que el paciente interactúe con el entorno de forma que no genere problemas. En el análisis de soluciones para $\mathrm{B}$ el paciente decidió el cambio de la conducta propia ya que esta es la que media la conducta con respecto al bajo rendimiento académico. De este modo, si logra desarrollar estrategias que le permitan adaptarse a los métodos y prácticas de enseñanza podrá interactuar de forma adecuada obteniendo el rendimiento académico esperado.

\section{Selección y diseño de estrategias para una futura intervención}

Para la selección y diseño de estrategias de intervención se consideró el planteamiento del análisis contingencial adicionando técnicas conductuales y considerando las respectivas funciones del terapeuta como agente activo en la intervención para el cambio (12). Cabe indicar que actualmente se están realizando estudios para incluir las técnicas de las terapias cognitivas de segunda y tercera generación pero aún no se ha verificado su eficiente aplicación, por lo que no serán incluidas en el esquema propuesto (13). 
Para lograr que el sujeto al interactuar disminuya sus dificultades para expresar y controlar sus emociones de forma adecuada, reduciendo la ira o la actitud pasiva, se aplicarán los procedimientos de desarrollo de competencias. Los cuales implican el desarrollo de habilidades para expresarse adecuadamente y controlar las emociones no deseadas. A tal fin se plantea usar las técnicas de entrenamiento asertivo y autocontrol. Las funciones interventoras que cumple el terapeuta son entrenar, instruir e instigar (12). Además modificar la conducta de la madre con el procedimiento de conducta de mediadores. Es decir, de las actitudes y conductas que influirán en aquellas no deseadas de la madre y/o pares como los insultos o gritos utilizando la técnica de administración de contingencias que le permitan en la interacción el incremento de las conductas esperadas.

Por otro lado, para que el paciente desarrolle conductas productivas en la interacción con el docente que permitan mejorar el rendimiento académico en matemáticas, se acordó modificar la conducta a través del desarrollo de competencias. Estos procedimientos van a permitir que el paciente cambie la conducta que media la interacción problemática como son la evitación, el mal humor, el sentimiento de frustración frente a los métodos y prácticas de enseñanza del docente (12). Este cambio se realizará con la técnica de modelamiento en la cual se le instiga al paciente a modelar la conducta para el desarrollo progresiva de conductas adaptativas y ensayo conductual. De este modo se logrará un desempeño conductual óptimo en actividades relacionadas a las matemáticas.

A nivel actitudinal se acordará con los padres y docente evitar las críticas destructivas a fin de modificar la relación y la percepción que puede tener el paciente de los mediadores. Esto a través del entrenamiento asertivo.

\section{CONCLUSIONES}

En el presente trabajo se propone el diseño de evaluación e intervención de un caso clínico a través de la metodología del análisis contingencial. Este modelo puede ser útil en la evaluación e intervención psicológica en adolescentes considerando que la mayor parte de la literatura publicada corresponde a intervenciones clínicas en adultos. El análisis contingencial propone diseñar la intervención en función de las interacciones del sujeto considerando a estas como las situaciones problemáticas, a diferencia de otros modelos que valoran como problema principal solamente la conducta del sujeto. El planteamiento del diseño de evaluación e intervención toma en cuenta criterios relacionados a la interacción y la valoración del problema. La aplicación del diseño propuesto permitiría, que ambos, terapeuta y paciente, asuman un papel activo en la solución de la situación problemática. Finalmente, se debe añadir, que el enfoque interconductual podría incorporar técnicas conductuales tradicionales.

\section{REFERENCIAS BIBLIOGRÁFICAS}

1. De Lourdes M, Landa P. El Análisis Contingencial: una nueva orientación para la psicología aplicable.UNAM, México; 1986.

2. Ribes E, López F. Teoría de la conducta. Un análisis de campo y paramétrico. México: Trillas; 1985.

3. Campuzano M. Mediación de relaciones de pareja. Un caso clínico desde el análisis contingencial. Revista Electrónica de Psicología Iztacala. 2010; 13(3).

4. Campuzano M, Rojo M, Jiménez, R. M. Problemas relacionados con la edad: un caso clínico, 2004.

5. Bernal C. La evaluación del aprendizaje escolar: una propuesta desde la psicología interconductual. Acta Comportamentalia: Revista Latina de Análisis de 1 Comportamiento, 2005.
6. Seañez M. La Psicología interconductual: un nuevo paradigma aplicado a la educación, 2007.

7. Bernal C. Diseño curricular basado en competencias profesionales: Una propuesta desde la Psicología interconductual. Revista de educación y desarrollo. 2007; 6(2):45-54.

8. Proust M. Psicología organizacional: consideraciones generales, 2002.

9. Campuzano M, DíazA. Competencias Laborales. Algunas propuestas. Enseñanza e investigación en Psicología. 2007; 12(1): 93-112.

10. De Lourdes M, Díaz A. La psicología en el escenario del Trabajo: Una revisión. Revista electrónica de psicología Iztacala. 2005; 8(2):45-57.
11. Vargas J. Psicología clínica: Intervención interconductual en el contexto clínico (IICC). Revista electrónica de Psicología Iztacala.2009; 6(2):23-35.

12. González E, Landa P, de Lourdes M, Ribes E, Sánchez, S. Análisis funcional de las terapias conductuales: una clasificación tentativa. Revista Española de Terapia del Comportamiento. 1989; 7(3):5466.

13. Patrón F. Análisis contingencial y el uso de técnicas cognitivo conductuales de segunda y tercera generación. Cathedra et Scientia. International Journal. 2016; 2(1):5379. 


\section{Contingency analysis: a proposal of intervention in a clinical case}

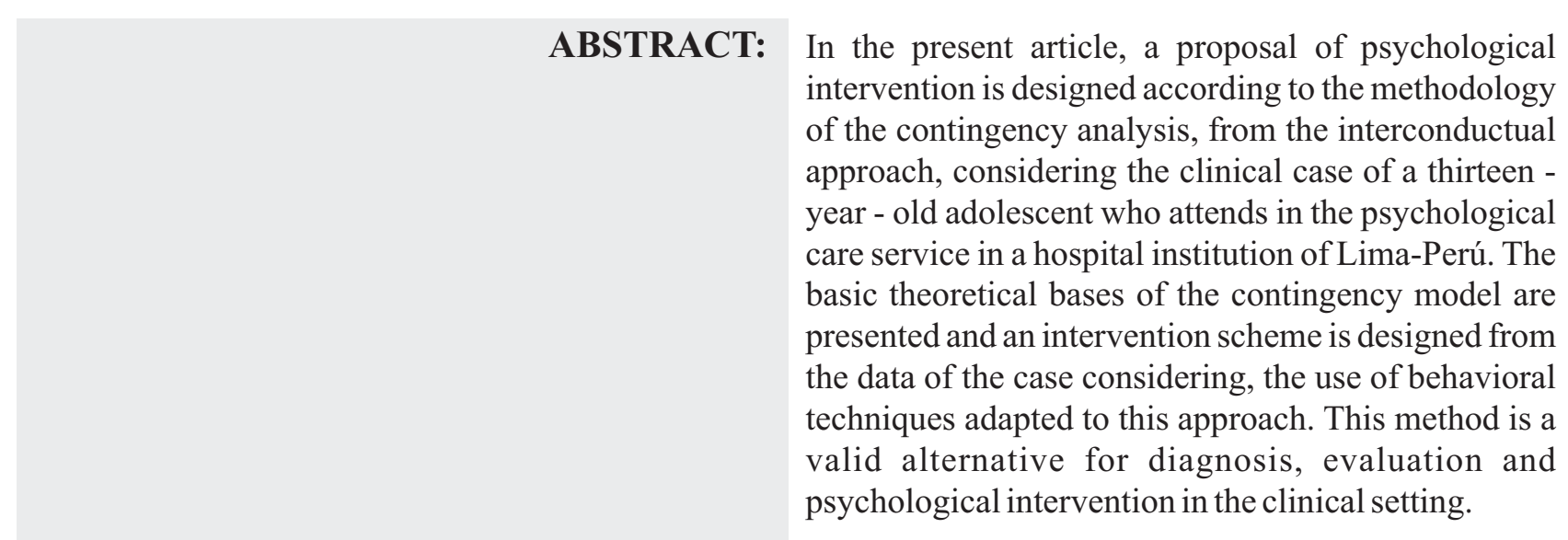

KEY WORDS: Contingency analysis; Interconductual approach; Clinical case, Adolescence 